\title{
Physikalische Therapie: Therapiemittel Elektrizität (2)
}

\author{
Jean-Michel Jeannin
}

Basel, Schweiz

\section{Einleitung}

In der vorliegenden Folge werden im Anschluss an den ersten Teil [1] weitere Anwendungsmöglichkeiten des physikalischen Therapiemittels Elektrizität zur Behandlung von psychiatrischen und neurologischen Störungen besprochen. Die hier vorgestellten Studien sind zur Illustration ausgewählt worden. Eine wissenschaftliche Bewertung von Publikationen und vorgestellten Methoden ist nicht Gegenstand des vorliegenden Beitrags.

\section{Craniale Elektrostimulation}

Unter der cranialen Elektrostimulation (CES) wird die Anwendung von niederfrequentem Wechselstrom über zwei auf der Kopfhaut oder den Ohrmuscheln angebrachten Elektroden verstanden. Hierzu wird Wechselstrom mit einer Frequenz von 0,5-100 Hz und einer Spannung von wenigen Volt mithilfe eines speziellen Geräts appliziert, das sich auch zur Selbstanwendung eignet und von der Food and Drug Administration (FDA) zugelassen ist. Die CES wird in der Schulmedizin eingesetzt, ist aber umstritten, da die Daten aus den publizierten Studien die strengen Kriterien nicht durchgehend erfüllen [2]. In der Praxis findet die CES eine vielfältige Anwendung: Sie wird zur Behandlung von bipolaren Depressionen, Schlafstörungen, posttraumatischen Belastungsstörungen, Angststörungen sowie chronischen Schmerzen eingesetzt $[3,4]$. Die CES wurde vor dem Durchbruch der medikamentösen Behandlung von Depressionen und Angstzuständen in Russland und den USA entwickelt und angewendet [2]. Als Wirkungsmechanismus wird eine direkte Einwirkung auf das limbische System, das aufsteigende retikuläre System und den Hypothalamus vorgeschlagen [5]. Shealy et al. [6] konnten nach einer 20 min dauernden Behandlung von gesunden Freiwilligen mit CES ein Ansteigen von Betaendorphin um 98\% im Plasma und um 219\% in der Cerebrospinalflüssigkeit sowie des Serotonins um 200\%
Abb. 1. Selene (lat. Luna), die Beschützerin der Heilkunst (Vatikanische Museen, Rom, 2016).

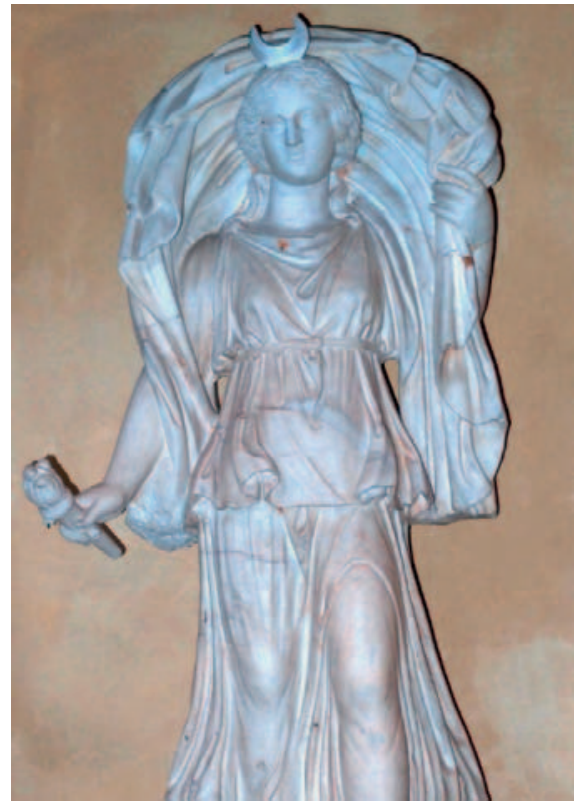

in der Cerebrospinalflüssigkeit beobachten. Das erklärt zumindest ansatzweise, weshalb die CES als Alternative zu einer medikamentösen Behandlung infrage kommt (Abb. 1). Im Rahmen der Postmarketing Surveillance wurden die Wirksamkeit und Sicherheit einer Selbstbehandlung von Angehörigen der US-Armee mittels Fragebogen ausgewertet. Von 1514 angefragten Personen sandten 145 einen auswertbaren Fragebogen zurück. Eine Verbesserung von mindestens $25 \%$ gaben $66,7 \%$ der Patienten an, die CES für eine Angststörung anwandten, sowie $65,3 \%$ der Patienten, die sie für eine Schlafstörung einsetzten, und 53,9\% der Patienten, die sie wegen einer Depression anwandten. 99\% der Antwortenden bewerteten die CES als sicher [3]. In einer Placebo-gesteuerten Studie mit 115 Patienten erwies sich eine 5 Wochen dauernde Behandlung mit CES gegenüber Placebo bezüglich der Angststörung und der Depression als statistisch signifikant überlegen. Es wurde von keinerlei unerwünschten Wirkungen berichtet [4]. 


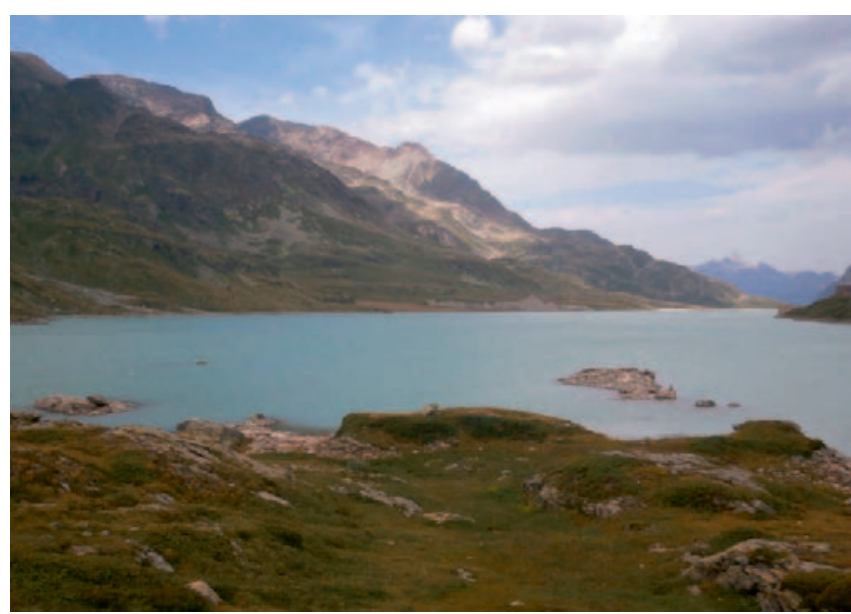

Abb. 2. Hier entsteht elektrischer Strom (Stausee, Bernina GR, 2013).

\section{Elektrotherapie von Schmerzen}

Die periphere diabetische Neuropathie ist eine schmerzhafte Komplikation des Diabetes. In einer kontrollierten randomisierten Studie behandelten Kumar und Marshall [7] Patienten, die an einer peripheren diabetischen Neuropathie litten, entweder mit transkutaner Elektrotherapie oder mit einer Scheinbehandlung. Beim applizierten Strom handelte es sich um biphasischen pulsförmigen Strom, dessen Frequenz vom Patienten zwischen 2 und $70 \mathrm{~Hz}$ angepasst werden konnte. Die Applikation des Stroms erfolgte mit einem Gerät. In der Behandeltengruppe $(\mathrm{n}=18)$ waren die Elektroden aktiv, in der Kontrollgruppe $(\mathrm{n}=13)$ inaktiv. Den Patienten wurde erklärt, dass sie den Strom nicht zwingend spüren müssen. In der Kontrollgruppe verzeichneten 5 Patienten eine statistisch signifikante Besserung der Symptome. Die Autoren vermuteten einen Effekt durch das Anwendungsverfahren. Alle gemessenen Parameter hatten sich bei der Behandeltengruppe statistisch signifikant gebessert (Symptomreduktion $52 \pm 7 \%$ vs. $27 \pm 10 \%$ ). Die Autoren sehen in der transkutanen Elektrostimulation eine wirksame nichtmedikamentöse Behandlung [7].

Eine Elektrotherapie kann auch Bestandteil einer physikalischen Komplextherapie sein (Abb. 2): Eine 36-jährige Frau leidet an stärksten Schmerzen am Schulterblatt mit Ausstrahlung bis in den Arm. Ursache ist ein Bandscheibenvorfall mit Kompression einer Nervenwurzel. Neben Analgetika und Antiphlogistika wird auch eine physikalische Komplextherapie verordnet, die Physiotherapie, Massnahmen der Detonisierung und eine Ultrareizstrom-Therapie nach Träbert umfasst. Die Schmerzen im Arm klingen innerhalb von 3 Wochen vollständig ab, die Elektrotherapie wird abgesetzt. Nach weiteren 3 Wochen mit Fortführung der übrigen physikalisch-medizinischen Massnahmen ist die Patientin beschwerdefrei [8].

\section{Reflexionen}

Gilula und Kirch [5] beschreiben die CES für die Behandlung von Depressionen als «sicherere Alternative zu Psychopharmaka». Kumar und Marshall [7] untersuchten Daten zur Wirksamkeit der sechs am meisten verschriebenen Antidepressiva, die der FDA im Zusammenhang mit dem Gesuch um Zulassung zwischen 1987 und 1999 vorgelegt wurden. Ungefähr $80 \%$ der Wirksamkeit der Medikamente wurden auch mit der Placebo-Behandlung erreicht. Der absolute Unterschied zwischen Medikament und Placebo betrug jeweils 2 Punkte sowohl auf der 50 Punkte als auch auf der 62 Punkte umfassenden Hamilton-Skala. Die Verbesserung war nach der höchsten Dosis nicht grösser als nach der niedrigsten Dosis. In einer Metaanalyse von 8 Studien mit insgesamt 1075 Patienten errechneten Kirsch und Smith (zitiert in [5]) einen Beitrag an die Gesamtwirksamkeit von 63\% einer CES-Behandlung versus 37\% der jeweiligen Kontrollbehandlung (Placebo + Verum $=100 \%)$. Mit der gleichen Berechnungsweise errechneten die Autoren einen Beitrag an die Gesamtwirksamkeit des Verums von 21\% versus $79 \%$ des jeweiligen Placebos. Es vermag zu Irritationen führen, dass die Anwendung von solchen Medikamenten als rational, d.h. vernünftig, gilt, jedoch die Anwendung eines Wechselstroms mit einer nicht wahrnehmbaren Stärke abgelehnt wird, weil die Wirksamkeit der Methode bisher noch nicht im selben Umfang wie jene von Medikamenten statistisch belegt nachgewiesen wurde. Nach Kavirajan et al. [9] fehlen vor allem Studien von hoher Qualität. Die Autoren verneinen aber die Wirksamkeit der Methode nicht. Pointiert formuliert, gilt es als vernünftig, für eine gegenüber Placebo nur wenig bessere Wirksamkeit eine ganze Reihe von Risiken in Kauf zu nehmen. Eine sehr risikoarme Behandlungsweise, wie es die CES nachweislich ist, wäre in der Tat eine prüfenswerte Alternative. Als Modalität der physikalischen Therapie weist die CES alle charakteristischen Aspekte auf: Die Anwendung ist mit Zuwendung verbunden (Arzt oder bei Selbstanwendung Patient); ausserdem ist sie ausgesprochen arm an Nebenwirkungen, nachhaltig und nicht zuletzt auch kostengünstig $[5,10]$.

\section{Schlussfolgerung}

Wichtige Momente therapeutischen Handelns sind mit den Methoden klinischer Studien nicht fassbar. In der Antike wurde die Medizin als Kunst betrachtet. In dieser Betrachtungsweise haben Intuition und feinste individuelle Abstimmung einer Therapie einen angemessenen Platz. 


\section{Literatur}

1 Jeannin JM: Physikalische Therapie: Therapiemittel Elektrizität. Schweiz Z Ganzheitsmed 2016;28:1-3.

2 Craniale Elektrostimulation. https://de.wikipedia. org/wiki/Craniale_Elektrostimulation.

3 Kirsch DL, et al: Military service member and veteran self reports of efficacy of cranial electrotherapy stimulation for anxiety, posttraumatic stress disorder, insomnia, and depression. US Army Med Dep J 2014;46-54.

4 Barclay TH, Barclay RD: A clinical trial of cranial electrotherapy stimulation for anxiety and comorbid depression. J Affect Disord 2014;164:171-177.
5 Gilula M, Kirch L: Cranial electrotherapy stimulation review: a safer alternative to psychopharmaceuticals in the treatment of depression. J Neurotherapy 2005;9:7-25.

6 Shealy CN, et al: Cerebrospinal fluid and plasma neurochemicals: response to cranial electrical stimulation. J Neurol Orthop Med Surg 1998; 18:94-97.

7 Kumar D, Marshall H: Diabetic neuropathy: amelioration of pain with transcutaneous electrostimulation. Diabetes Care 1997;20: 1702-1705.
8 Glaesener JJ: 3.7 Elektro- und Ultraschalltherapie; in Gutenbrunner C, Glaesener JJ: Rehabilitation, Physikalische Medizin und Heilverfahren. Heidelberg, Springer, 2007, pp 69.

9 Kavirajan HC, et al: Alternating current cranial electrotherapy stimulation (CES) for depression. Cochrane Database Syst Rev 2014; 7:CD010521.

10 Kirsch I, Moore TJ: The emperor's new drugs: an analysis of antidepressant medication data submitted to the U.S. Food and Drug Administration. Prevention and Treatment 2002;5 Article 23. http://psycnet.apa.org/journals/ pre/5/1/23a/. 